University of Nebraska - Lincoln

DigitalCommons@University of Nebraska - Lincoln

$1-19-2008$

\title{
Different approaches to adjusting band offsets at intermolecular interfaces
}

Peter A. Dowben

University of Nebraska-Lincoln, pdowben@unl.edu

Jie Xiao

University of Nebraska-Lincoln, jie.xiao@helmholtz-berlin.de

Bo Xu

University of Nebraska-Lincoln

Andrei Sokolov

University of Nebraska-Lincoln, sokolov@unl.edu

Bernard Doudin

Institut de Physique Applique de Physique et Chimie des Materiaux de Strasbourg, 23 rue du Loess, B.P. 20,67034 Strasbourg Cedex 2, France, bernard.doudin@ipcms.unistra.fr

Follow this and additional works at: https://digitalcommons.unl.edu/physicsdowben

Part of the Physics Commons

Dowben, Peter A.; Xiao, Jie; Xu, Bo; Sokolov, Andrei; and Doudin, Bernard, "Different approaches to adjusting band offsets at intermolecular interfaces" (2008). Peter Dowben Publications. 204.

https://digitalcommons.unl.edu/physicsdowben/204

This Article is brought to you for free and open access by the Research Papers in Physics and Astronomy at DigitalCommons@University of Nebraska - Lincoln. It has been accepted for inclusion in Peter Dowben Publications by an authorized administrator of DigitalCommons@University of Nebraska - Lincoln. 
Published in Applied Surface Science (2008); doi:10.1016/j.apsusc.2008.01.062

Copyright (C) 2008 Elsevier B.V. Used by permission. http://www.sciencedirect.com/science/journal/01694332

Published online January 19, 2008.

\title{
Different approaches to adjusting band offsets at intermolecular interfaces
}

\author{
P. A. Dowben ${ }^{1}$, Jie Xiao $^{1}, \mathrm{Bo} \mathrm{Xu}^{1,3}$, Andrei Sokolov ${ }^{1}$, and B. Doudin ${ }^{2}$ \\ ${ }^{1}$ Department of Physics and Astronomy, Nebraska Center for Materials and Nanoscience, \\ University of Nebraska-Lincoln, Lincoln, NE 68588-0111, USA \\ ${ }^{2}$ Institut de Physique Applique de Physique et Chimie des Materiaux de Strasbourg, \\ 23 rue du Loss, B.P. 20, 67034 Strasbourg Cedex 2, France \\ 3 Present address: State Key Laboratory of Metastable Materials Technology and Science, \\ Yanshan University, Qinhuangdao, Hebei, PR China \\ Corresponding author: P. A. Dowben, pdowben@unl.edu
}

\begin{abstract}
We show that at the hetero-molecular interface, the molecular band offsets can be modified by either adjusting the dopant or dopant concentration of one or both molecular layers or by changing the dipole orientation at the intermolecular interface. Photoemission studies reveal the changes in electronic structure and diode devices exhibit conduction properties that are altered in response to changes to the molecular band offsets. As a demonstration, thin film copper phthalocyanine to crystalline ferroelectric copolymer poly(vinylidene fluoride with trifluoroethylene) heterojunction diodes, without additional dopants, are compared with doped polyaniline layers on the crystalline ferroelectric copolymer poly(vinylidene fluoride with trifluoroethylene).
\end{abstract}

Keywords: organic heterojunctions, ferroelectric polymers, molecular orbital alignment

JEL classification codes: $71.20 . \mathrm{Rv} ;$ 68.35.Fx; 81.15.Ef; 85.30.Kk

\section{Introduction}

The addition of ligands, doping, or alloying necessary for significant chemical shifts of the valence band results in significant changes in the molecular orbitals (in molecules) and band structure (in solids) and can obscure changes in the valence band due to simple charge addition or subtraction. With insulators, doping can lead to a number of interesting complications. Changes in electron localization can lead to dramatic changes in photoemission screening [1] and, as a consequence, photoemission final state effects rather than initial state chemical shifts can dominate the valence band [2]. For wide band gap insulators, doping can lead to the formation of a density of states in the gap (Hubbard bands). These new bands can appear well away from the Fermi level (both above and below the Fermi level) as a result of the on site correlation energies. These Hubbard bands have been observed with the alkali metal doping of a number of molecular systems with a large gap between the highest occupied molecular orbital (HOMO) and the lowest unoccupied molecular orbital (LUMO) $[1,3,4]$. If the molecular orbitals are sufficiently delocalized, organic conductors provide an avenue for the direct investigation of systematic shifts of the valence and conduction band states as a result of charge transfer.

The role of interface dipoles in heterojunction devices using semiconducting organics (as basic constituent for organic electronics applications) resides on two key issues: charge injection and the molecular band offsets. For many large molecular adlayers, including a number of organic and metal-organic species, the energy level alignment (of the adsorbate) is dependent upon the interfacial electronic structure and the interfacial dipole layer, as has been readily demonstrated for many large molecules [5-7], including the metal phthalocyanines [8]. Charge injection and the dipoles at the electrode interface are surprisingly complex [9] because of interface chemistry. At the interface between the two organic layers, the expected weaker chemical interactions permit better understanding of the molecular band offsets, in spite of usually limited understanding of the in- 
terface structure. This weak interaction between the metal phthalocyanines and an organic polymer substrate should, in fact, be weaker than the interactions observed at insulating substrate, where the latter interactions are seen to be less strong than for a conducting substrate [10].

The obvious way to control interface dipoles is to use a semiconducting ferroelectric thin films. Organic ferroelectrics are among the best candidates for such studies as with such systems band bending is minimized and one can ensure compositional control of the interface. Organic ferroelectrics have been exploited as components in heterojunction devices, in particular using the better known [11] copolymers of the ferroelectric copolymer poly(vinylidene fluoride with trifluoroethylene) (P(VDF-TrFE)). Organic heterojunction diodes [12, 13] and transistors $[14,15]$ have been fabricated with the crystalline ferroelectric P(VDFTrFE). As the copolymer poly(vinylidene fluoride with trifluoroethylene) is a ferroelectric, transistors, both fully organic heterojunctions [14] and [15] and hybrid heterojunctions with an inorganic $\mathrm{SiO}_{2}$ buffer layer integrated state gate over silicon $[16,17]$, exhibit gate voltage-dependent hysteresis, indicating the potential of P(VDF-TrFE) copolymers for nonvolatile random access memory devices. Evidence for interface dipole coupling, however, is better demonstrated in the diode geometry, as described here.

Here we show how it is possible to design heterojunction devices involving organic thin films, of interface electronic properties tunable through doping or voltage control of interface dipoles. We present the reasoning leading to the choice of adequate organic films compatible with (inverse) photoemission studies (or ultra-high vacuum compatible). Such experimental techniques provide insight into the density of states around the Fermi level, and indicate how doping can allow tuning of the semiconducting properties. We present electrical characteristics of the basic device for applications, namely a heterojunction diode, as a confirmation of the expected and desired properties for use in organic electronics applications.

\section{Some background on the organic semiconduc- tors compared}

Both polyaniline [12, 18-21] and copper phthalocyanine (CuPc) thin films $[8,13,22-27]$ (schematically illustrated in Figure 5) can be produced by vapor deposition compatible with ultrahigh vacuum photoemission. These films can be grown very thin (100 $\AA$ or less) and are free of solvent contamination. Such films exhibit a perpendicular resistance of only a few ohms at temperatures as low as $4 \mathrm{~K}$ and are thus free of surface charging in photoemission.

Copper phthalocyanine, like polyaniline, is an organic semiconductor and has been used in field effect devices $[28,29]$. Because of the small, adsorption induced, instantaneous dipole, copper phthalocyanine was chosen [13] for the organic heterostructure with P(VDF-TrFE) as a comparison with polyaniline $[12,21]$ rather than the more conventional organic semiconductors like poly(methoxy(ethylhexloxy)-p-phenylene-vinylene) (MEH-PPV) [14], or poly(ethyenedioxythiophene)-polystryene-sulphonic acid (PEDOT-PSS) [15].
Although copper phthalocyanine is considered a planar molecule with no permanent electric dipole [22], the molecular orbital alignment of adsorbed copper phthalocyanine is affected by both interface dipoles $[8,13]$ and molecular orientation $[13,22]$ while interactions or charge transfer influence the highest occupied (HOMO) to lowest unoccupied (LUMO) gap [23]. Indeed, with adsorption on metallic substrates copper phthalocyanine exhibits an electric dipole as the molecular center copper atom is not in the same plane as the surrounding ligand, and adopts a position that is typically closer to the interface [27]. The changes in the copper phthalocyanine orientation and dipole make this metalorganic semiconductor a useful adsorbate for the study of effects of dipole interactions at a heterojunction interface [13]. While high dielectric constant composites of CuPc have been fabricated with $\mathrm{P}(\mathrm{VDF}-\mathrm{TrFE})$ and like copolymers [30, 31], the structures considered here are that of an organic heterojunction in the mesa geometry.

\section{Experiment}

The polyaniline samples were prepared by vacuum deposition on Au-coated (100 nm) silicon substrates in a preparation chamber vacuum continuous with the spectrometer vacuum systems $[12,13]$. A SEAS Na getter was used to dope the polyaniline films with sodium, while iodine vapor was used to introduce iodine into the films. The Na $2 \mathrm{~s}$ to $\mathrm{C}$ 1s X-ray photoemission (XPS) intensity ratios show no emission angle dependence following $\mathrm{Na}$ deposition, or iodine adsorption indicating the sodium is uniformly distributed in the surface region of vapor deposited polyaniline.

The band positions, relative to the Fermi level, of P(VDFTrFE) films and pure/doped polyaniline covered P(VDFTrFE) films, with each polymer about five monolayers thick, were determined through inverse photoemission (IPES) and photoemission (PES), undertaken in the same UHV chamber, as described elsewhere $[12,13,20]$. The CuPc thin films, of $2 \mathrm{~nm}$ thickness and greater, were also evaporated onto $\mathrm{Au}$ and $\mathrm{P}(\mathrm{VDF}-\mathrm{TrFE})$ substrates (the latter also deposited on $\mathrm{Au}$ and graphite) in a preparation chamber, vacuum continuous with the spectroscopy ultra-high vacuum (UHV) chamber, at a rate of about $0.2 \mathrm{~nm} / \mathrm{min}$ [13]. Combined photoemission and inverse photoemission spectra were taken of 2-nm thick $\mathrm{CuPc}$ films (nominally five monolayers [28]) and nominally five monolayers thick P(VDF-TrFE) copolymer "bottom" films on graphite (roughly $2-3-\mathrm{nm}$ thick films) and $\mathrm{Au}\left(\begin{array}{lll}1 & 1 & 1\end{array}\right)$.

For the perpendicular transport measurements, mesa structures were fabricated by growing nominally 30 monolayers (about $1.7 \mathrm{~nm} /$ monolayer) of $\mathrm{P}(\mathrm{VDF}-\mathrm{TrFE})$ copolymer films used as substrates for the vapor deposited polyaniline thin films $[12,19-21]$ with polyaniline polymer films about 80 molecules $(20 \mathrm{~nm})$ thick, or $2-4 \mathrm{~nm}$ of $\mathrm{CuPc}$ on $2-3 \mathrm{~nm}$ of $\mathrm{P}(\mathrm{VDF}-\mathrm{TrFE})$. The thin films thickness was dictated by a need to ensure layers free of pin-holes while still as thin as possible to diminish contributions of final state photoemission effects. Throughout, the P(VDF-TrFE) thin films were made on $\mathrm{Al}, \mathrm{Au}$ and graphite substrates using the Langmuir-Blodgett (LB) technique, as described elsewhere $[11,12]$, and are seen to be highly crystalline on graphite substrates [32-34]. 
The angle-resolved photoemission spectroscopy experiments were performed on the $3 \mathrm{~m}$ toroidal grating monochromator at the Center for Advanced Microstructure and Devices (CAMD) [35]. The angle-resolved photoemission spectra reproduced here were taken with photon energy of $70 \mathrm{eV}$, unless otherwise stated, the photon incident angle was $40^{\circ}$ from the surface normal and the photoelectrons were collected normal to the surface. While P(VDF-TrFE) does exhibit band structure of the unoccupied bands [32, 36 , 37], the occupied bands exhibit only a small band width and little dispersion with wave vector $\left(k_{||}\right)$[38], so that the angle-resolved photoemission studies were primarily undertaken to determine molecular orientation as described elsewhere [39]. The He I (21.2 eV) ultraviolet photoemission (UPS), angle-resolved inverse photoemission, and XPS were undertaken in a single UHV chamber $[12,13,21]$. Photoemission in this latter instrument was effectively angle-integrated as photoelectrons were collected from a solid angle of $\pm 10^{\circ}$ or more. The Fermi level was determined from a tantalum foil or a gold substrate in intimate contact with the sample substrate. The combination of photoemission and inverse photoemission permits some assessment of the highest occupied to lowest unoccupied molecular orbital gap as well as the molecular band offsets, when carried out in the fashion described here [39]. Inverse photoemission probes the unoccupied molecular orbitals without the elemental specificity of Xray absorption spectroscopies [40], but is easier to reference to a common Fermi level and does not suffer from the energy perturbations introduced by the excitation hole, as occurs in some electron energy and characteristic energy loss spectroscopies.

\section{Charge filling and depletion}

The influence of electron donation from increasing concentrations of sodium, as well as electron subtraction from iodine doping of vapor deposited polyaniline on gold is shown in Figure 1. Both the occupied and unoccupied molecular orbitals of the freshly evaporated polyaniline thin films shift to greater or smaller binding energies (with respect to the Fermi level) when doped with sodium or iodine, respectively [21]. This is very different from sodiumdoped poly(p-pyridine) [41] and other polymeric systems $[1,4]$, where sodium-doping induces new states in the otherwise empty band gap.

As seen in Figure 1, the shifts in binding energies for valence bands or occupied molecular orbitals (features AC) are the same and while the unoccupied levels (feature D) appear to exhibit a smaller shift in binding energy, the trend is very similar. Several fitting routines, with one illustrated in Figure 1, were used to ascertain the shifts in the unoccupied levels, and all provided similar results [21]. The labeled occupied molecular orbital features in photoemission are dominated by molecular orbitals like that shown in the inset to Figure 1 [19-21].

In a band-filling picture, introducing extra electrons to the polyaniline system, through sodium atom doping, fills any empty states near the Fermi level and increases the binding energies of all the molecular orbitals. The subtraction

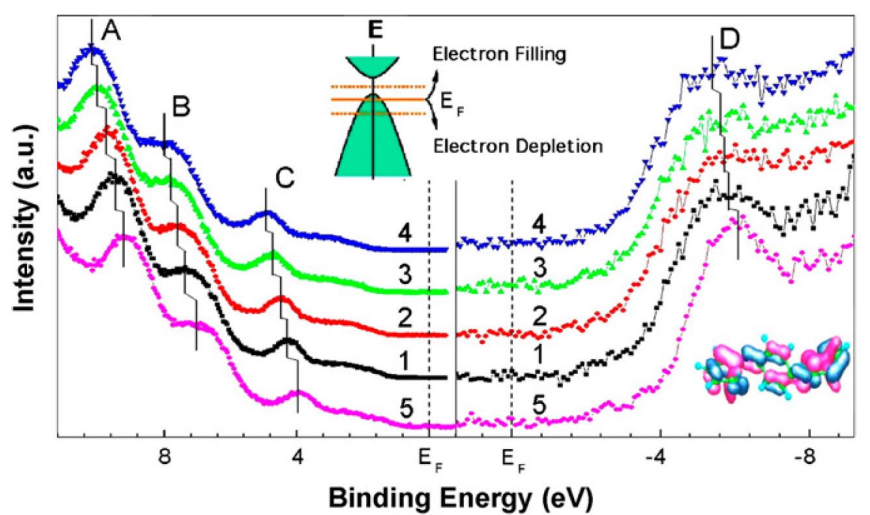

Figure 1. Angle-integrated ultraviolet photoemission spectra (left) and inverse photoemission spectra (right) (at normal incidence) of freshly evaporated polyaniline thin film (100 ̊̊) as well as following sodium and iodine doping. Spectrum 1 is from a clean, as deposited, polyaniline film, spectra 2-4 are from sodium-doped film with increasing sodium exposure while spectrum 5 is from iodine-doped film. Three photoemission features, and one unoccupied inverse photoemission feature show similar binding energy shifts with doping (denoted as features: A-D, respectively). The labeled occupied molecular orbital features in photoemission are dominated by molecules orbitals [19-21] like that shown in the inset. The schematic of electron filling and depletion is shown as a separate insert.

of electrons from the polyaniline system by doping with iodine atoms leads to a decrease in binding energy of all the bands. This concept is schematically indicated in the inset of Figure 1. For films both thinner $(\sim 50 \AA)$ and thicker $(\sim 200 \AA)$ than those shown in Figure 1, the shifts are qualitatively similar, but not as clearly evident. In the former case, this is due to the higher density of states and in the latter case, it becomes increasingly difficult to dope the film uniformly to significant levels by the methods employed here. As noted in Section 3, the dopant atoms do not form a surface layer, but are isotropically distributed in the surface region probed by photoemission and inverse photoemission: this is also clear from the absence of dramatic changes in the photoemission peak widths with doping (Figure 1) as well as the angle-resolved XPS measurements.

All the molecular orbitals (both occupied and unoccupied) shift, to greater (sodium) or smaller (iodine) binding energies with respect to the Fermi level by very similar amounts with doping. Therefore, the molecular orbitals shifts are dominated by charge transfer in the initial state and not by final state effects [2]. The suppression of final state effects, in spite of changes in conductivity and the density of states in the vicinity of the Fermi level associated with such kinds of doping, suggests that the molecular orbitals are delocalized in polyaniline.

Since vapor deposited polyaniline is a p-type semiconductor, the simple picture of band filling or depletion explains why doping the material with electron donors makes this (and similar conducting polymers) more insulating, while doping with electron acceptors (halogens, perchlorate and the like) substantially increases conductivity, as is generally accepted [42]. In the former case, the Fermi level is in a gap of the band structure, while in the latter case, the Fermi level resides well within an appreciable density of states of the band structure. 


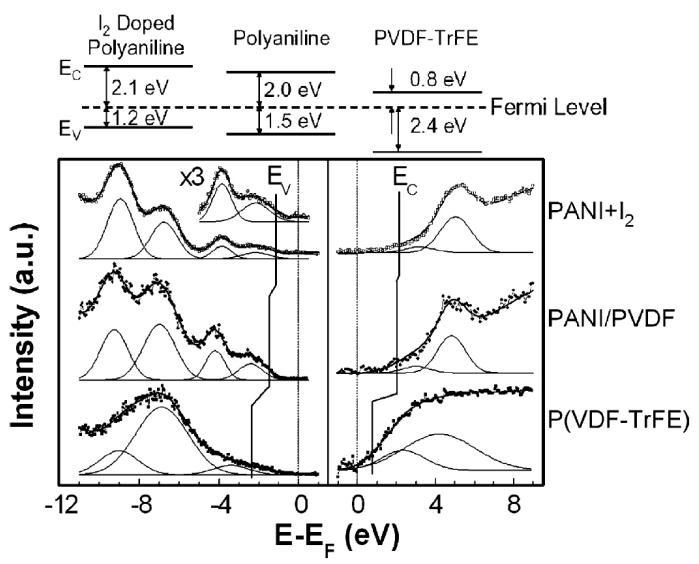

Figure 2. Photoemission and inverse photoemission spectroscopy of clean $(\mathrm{P}(\mathrm{VDF}-\mathrm{TrFE})$ ) and polyaniline covered (PANI) $\mathrm{P}(\mathrm{VDF}-\mathrm{TrFE})$ films as well as iodine-doped polyaniline (PANI $\left.+\mathrm{I}_{2}\right)$. The spectra are fitted using Gaussian functions and the relative shifts in the edges of valence band and conduction band are shown in the figure using vertical bars. The corresponding energy levels of these components are schematic shown at the top of the figure.

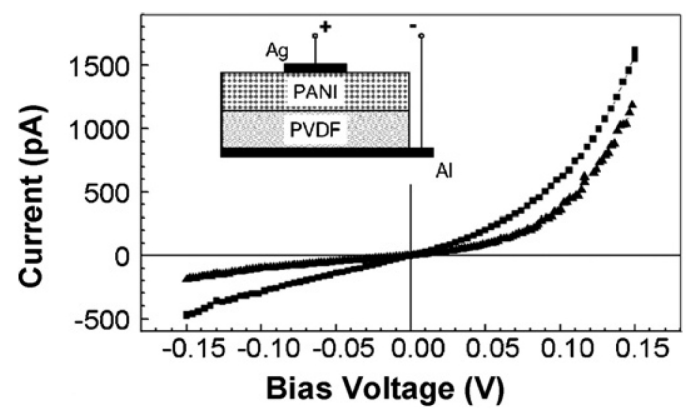

Figure 3. $I-V$ characteristics of diodes fabricated from $\mathrm{P}(\mathrm{VDF}-\mathrm{TrFE})$ ( $30 \mathrm{Ml}$ thick) and polyaniline (200 $\AA$ or 80 monolayers thick). Two different kinds of polyaniline, pristine as well as iodine-doped, were used in the diode fabrication. The corresponding room temperature $I-$ $V$ curves are shown, with $(\mathbf{\square})$ and $(\boldsymbol{\Lambda})$ for pristine and iodine-doped polyaniline, respectively.

From the position of the HOMO and LUMO states and the position of the valence and conduction band edges, as determined by photoemission and inverse photoemission at room temperature (Figure 2), the ferroelectric P(VDFTrFE) may be considered simply a good insulator or a weakly n-type semiconductor, based on the placement of the Fermi level closer to the conduction band edge (the lowest unoccupied molecular orbitals), although the type of majority carrier has not been measured. Vapor deposited polyaniline is, however, generally of p-type character. Judging by the shift in molecular orbital positions relative to the Fermi level, doping with iodine leads to a decrease in binding energy in all the polyaniline bands [21], likely enhancing the p-type character of this polymeric semiconductor, as seen in Figure 1 (although the caveat that the majority carrier has not been directly determined). Because of the band offsets, schematically illustrated in Figure 2, it is nonetheless possible to fabricate a heterojunction diode with a P(VDF-TrFE) and polyaniline (both doped and undoped) bilayer [12].
Heterojunction $\mathrm{p}-\mathrm{n}$ diode structures from $\mathrm{P}(\mathrm{VDF}-\mathrm{TrFE})$ and polyaniline, schematically shown as an insert in Figure 3 , indeed exhibit excellent diode characteristics, when suitably doped. Rectification is evident in the $I-V$ curves of two diodes, both from a $\mathrm{P}(\mathrm{VDF}-\mathrm{TrFE}) /$ polyaniline bilayer and $\mathrm{P}(\mathrm{VDF}-\mathrm{TrFE})$ with polyaniline doped with iodine, as shown in Figure 3. With iodine doping, the polyaniline becomes more p-type and the density of minority charge carrier decreases, resulting in a diode with overall better performance, based on the reverse bias current. From the shift in the Fermi level relative to the conduction band edge, we expect that the resulting reverse bias current should decrease for the $\mathrm{p}-\mathrm{n}$ junction fabricated from iodine-doped polyaniline, compared to the undoped (and less p-type) polyaniline, as is indeed observed in the experiment.

\section{Interface dipole alignment}

While doping is a (more) conventional method for shifting the molecular orbital band offsets with respect to the Fermi level, changing molecular orbital alignment can be undertaken in other ways. One approach is to change the interface dipole alignment. This has been demonstrated for thin film copper phthalocyanine to crystalline ferroelectric copolymer poly(vinylidene fluoride with trifluoroethylene) heterojunction diodes [13].

At the outset, it is not necessarily clear from the photoemission and inverse photoemission of P(VDF-TrFE) and $\mathrm{CuPc}$ on gold that $\mathrm{CuPc}$ would make a reasonable heterojunction diode with $\mathrm{P}(\mathrm{VDF}-\mathrm{TrFE})$, although it does [13]. For $\mathrm{CuPc}$ deposited on $\mathrm{Au}$ [23] (Figure 4a), we found qualitative agreement with prior photoemission $[8,22-25]$ and inverse photoemission $[23,26]$ experimental data. The positions of the band edges, as determined by photoemission and inverse photoemission at room temperature, do not directly demonstrate that $\mathrm{CuPc}$ is strongly more p-type relative to $\mathrm{P}(\mathrm{VDF}-\mathrm{TrFE})$. The different positions of the valence and conductance band edges for CuPc vacuum deposited on P(VDF-TrFE) do, however, clearly indicate that it may be possible to construct a heterojunction diode from these two dissimilar organic molecular systems [13].

The origin of the huge differences in band gap and molecular orbital position between $\mathrm{CuPc}$ on $\mathrm{Au}$ and $\mathrm{CuPc}$ on $\mathrm{P}(\mathrm{VDF}-\mathrm{TrFE})$ indeed suggests that CuPc does not lie flat on $\mathrm{P}(\mathrm{VDF}-\mathrm{TrFE})$ [13]. The low-lying CuPc unoccupied molecular orbitals, along with the highest occupied molecular orbitals have strong $\mathrm{Cu}$ and $\mathrm{N}$ weight. Therefore, molecules lying well out of the plane of the surface are expected to have molecular orbitals with strong $\mathrm{Cu}$ and $\mathrm{N}$ weight suppressed in a highly surface sensitive spectroscopy like inverse photoemission while molecular orbitals with stronger ligand weight would not be so strongly suppressed, as observed in Figure 4. This is consistent with the fact that $\mathrm{CuPc}$ tends to lie flat on metal substrates and stand on oxide surfaces [43]. Regrettably, photoemission features alone are not an effective means of determining orientation and crystal packing of $\mathrm{CuPc}$, although this has been attempted [24], so further measurements such as light incident angle dependent near edge X-ray absorption fine structure measurements are indicated, but as yet, have not been carried out. 


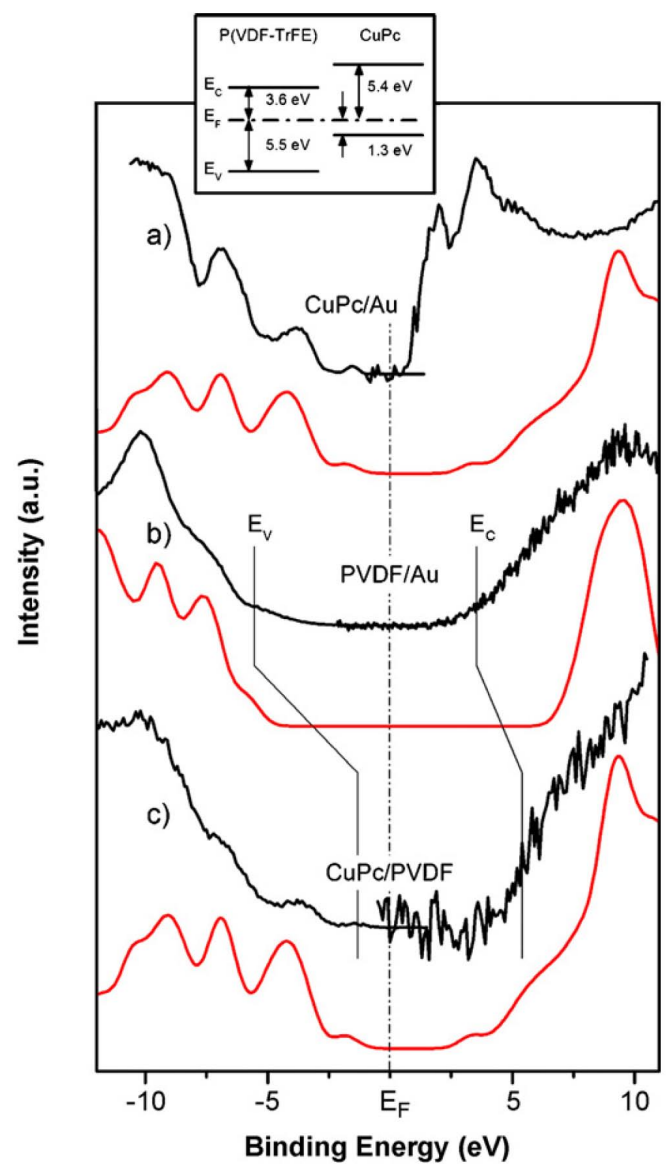

Figure 4. Photoemission and inverse photoemission spectra of poly(vinylidene fluoride with trifluoroethylene) on $\mathrm{Au}$ (b) and $\mathrm{CuPc}$ adsorbed on $\mathrm{Au}(\mathrm{a})$ and on the crystalline ferroelectric P(VDF-TrFE) (c). For comparison, a rough expectation of the ground state density of states is shown as derived from semiempirical single molecule calculations (PM3) with a $1 \mathrm{eV}$ Gaussian width added to the eigenvalues, with no correction for final state or matrix element effects (red curves). The relative shifts in the edges of valence band and conduction band are indicated by vertical bars for PVDF on Au and CuPc on PVDF on Au. The corresponding energy levels of these components are schematically shown in the inset, to indicate the relative band offsets of the two molecular systems, in the pertinent heterojunction.

Strong interactions or charge transfer to $\mathrm{CuPc}$ are believed to decrease the size of the HOMO-LUMO gap for $\mathrm{CuPc}$ inferred from combined photoemission and inverse photoemission spectra [23]. An increase in the HOMOLUMO gap, as observed for CuPc on P(VDF-TrFE), and the similarity of the HOMO-LUMO gap and the density of states with the semiempirical PM3 theory (Figure 4) suggests that the electronic structure of CuPc on P(VDF-TrFE) more closely resembles that of an isolated molecule, not the fully screened gap of an adsorbed molecule on a metal substrate.

Although we cannot ascertain the majority carrier for $\mathrm{CuPc}$ thin films from electron spectroscopies alone (i.e. is it n-type or p-type), the band alignment for CuPc on P(VDFTrFE) suggests that a heterojunction diode can be fabricated with $\mathrm{P}(\mathrm{VDF}-\mathrm{TrFE})$, as has been done for polyaniline and $\mathrm{P}(\mathrm{VDF}-\mathrm{TrFE})$, as discussed above. Using pre-templated Au bottom electrodes and evaporated Al top elec-

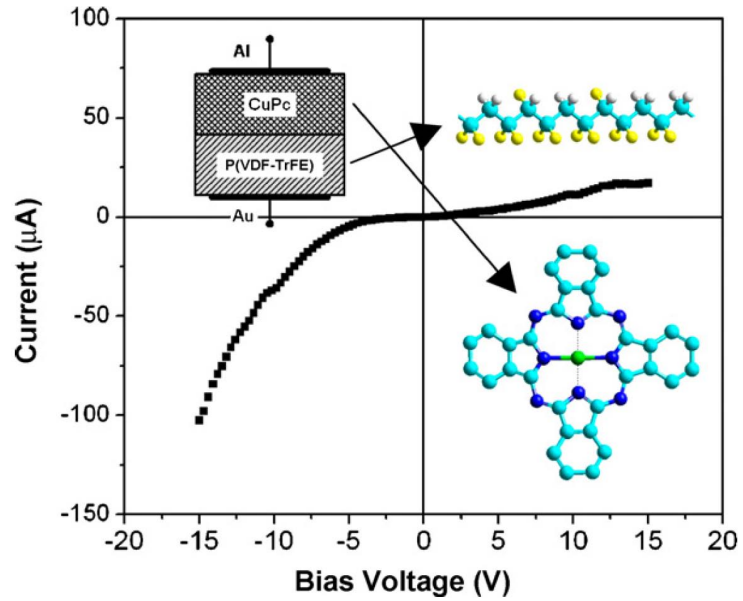

Figure 5. $I-V$ characteristics of diodes fabricated from $4 \mathrm{~nm}$ of $\mathrm{CuPc}$ deposited on $3 \mathrm{~nm}$ of P(VDF-TrFE), as indicated by the inset schematic structure. Schematic representations are also shown of P(VDFTrFE) (top) and CuPc (bottom), with carbon (blue), fluorine (yellow), nitrogen (navy blue), and copper (green) indicated. For CuPc, the hydrogen has been left off to simplify the schematic.

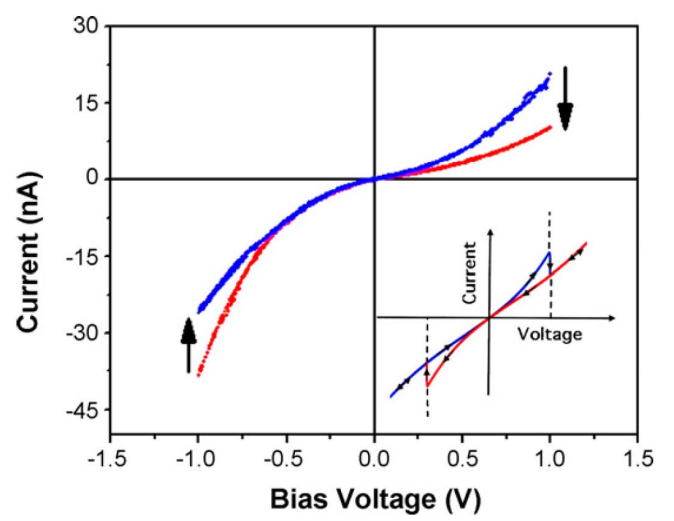

Figure 6. Reproducible current differences in the $I-V$ curves are seen at small bias in changing the applied voltage from -15 to $+15 \mathrm{~V}$ (blue) and +15 to $-15 \mathrm{~V}$ (red) in heterojunctions formed from $4 \mathrm{~nm}$ of $\mathrm{CuPc}$ deposited on $3 \mathrm{~nm}$ of P(VDF-TrFE). A schematic of the expectation $[47,50]$ for a ferroelectric tunnel junction barrier are indicated in the inset as adapted from [47].

trodes (to ensure ohmic contacts) such diodes were fabricated [13] using 3-4 $\mathrm{nm} \mathrm{P}(\mathrm{VDF}-\mathrm{TrFE})$ and $4 \mathrm{~nm} \mathrm{CuPc}$ deposited by vapor deposition, as seen in Figure 5 . The onset of rectification at $1-4 \mathrm{eV}$ for these diodes is consistent with the molecular orbital alignment inferred from Figure 4, with $\mathrm{P}(\mathrm{VDF}-\mathrm{TrFE})$ acting more n-type than CuPc.

Indications of dipole interaction between $\mathrm{CuPc}$ and ferroelectric material $\mathrm{P}(\mathrm{VDF}-\mathrm{TrFE})$ are evident in the electric field controlled $\mathrm{p}-\mathrm{n}$ diode formed by combining these two molecular thin films together. By biasing the diode by $\pm 15 \mathrm{~V}$, hysteresis is seen in the current versus voltage traces at low bias, as seen in Figure 6. Application of bias voltages up to $\pm 15 \mathrm{~V}$ is more than sufficient to polarize the ferroelectric $\mathrm{P}(\mathrm{VDF}-\mathrm{TrFE})$ [11], and/or flip the dipole direction of $\mathrm{P}(\mathrm{VDF}-\mathrm{TrFE})$ (or both $\mathrm{P}(\mathrm{VDF}-\mathrm{TrFE})$ and CuPc) thin films of this thickness, so this is not entirely unexpected. The dipole interaction could affect the diode properties in several ways. The local electric field due to the P(VDF-TrFE) could 
Dowben, Xiao, Xu, Sokolov, \& Doudin in Applied Surface Science (2008)

align the CuPc's dipoles and either change the molecular orientation(s) or change the molecular dipole alignment(s) at the interface thus leading to a decrease or increase in the barriers to current. Changes in the barrier height can have dramatic changes in the tunneling current $[44,45]$, which cannot be completely excluded, as both organic films are quite thin (to exclude final state effects and charging). Expectations on what should be the behavior of a ferroelectric tunnel junction barrier, based on the Brickman model [46], have been much discussed [47-50], and these expectations are borne out in the $\mathrm{P}(\mathrm{VDF}-\mathrm{TrFE})-\mathrm{CuPc}$ heterojunction device, as indicated in Figure 6. Other ferroelectric tunnel junction devices, using inorganic ferroelectric barrier layers, also show hysteresis but the hysteresis for the $\mathrm{P}(\mathrm{VDF}-\mathrm{TrFE})-\mathrm{CuPc}$ heterojunction device is reversed from some [47, 48, 51, 52], but not all [53] inorganic ferroelectric barrier layers reported elsewhere. The hysteretic current-voltage $(I-V)$ characteristics may differ from the $\mathrm{P}(\mathrm{VDF}-\mathrm{TrFE})-\mathrm{CuPc}$ heterojunction device shown here because of space-charge-limited-current conduction with the inorganic ferroelectric barrier layers [51].

Alternatively, changes in dipole orientation or dipole alignment can change the molecular orbital alignment of $\mathrm{CuPc}$ relative to $\mathrm{P}(\mathrm{VDF}-\mathrm{TrFE})$, resulting in an effective change in the "band offsets" and the diode characteristics. In the measurements we have undertaken here, both effects of the dipole interactions at the organic molecular interface would be similar and lead to hysteretic effects.

\section{Summary}

Organic heterojunctions provide new possibilities for interface control and engineered semiconducting properties. As an example of new functionality, we found that dipole interactions between CuPc and P(VDF-TrFE) play a key role in our combined photoemission and inverse photoemission spectra and such interface dipole interactions may be the origin of the electric field "controlled" diodes fabricated from CuPc and ferroelectric P(VDF-TrFE), the latter being a ferroelectric material with a strong intrinsic (reversible) dipoles. This effect was not seen in P(VDF-TrFE) to polyaniline heterostructures where the molecular band offsets were easily adjusted by doping. Our experimental strategy also illustrates how surface spectroscopy techniques provide information on the buried interface electronic properties, of direct relevance for designing and understanding electric transport properties of electronic devices.

\section{Acknowledgments}

This work was supported by the National Science Foundation through grant CHE-0415421, and CHE-0650453 and the NSF "QSPINS" MRSEC (DMR 0213808). The authors would also like to acknowledge the assistance, contributions as well as help conversations with Stephen Ducharme, Evgeny Tsymbal, Jaewu Choi, Y. Ovchenkov, A.V. Sorokin, Valdimir Fridkin, and Anthony Caruso.

\section{References}

[1] P. A. Dowben, Surf. Sci. Rep. 40 (2000), p. 151.

[2] J. E. Ortega, F.J. Himpsel, D. Li, and P. A. Dowben, Solid State Commun. 91 (1994), p. 807.

[3] D. N. McIlroy, C. Waldfried, T. McAvoy, J. Choi, P. A. Dowben, and D. Heskett, Chem. Phys. Lett. 264 (1997), p. 168.

[4] J. Choi, P. A. Dowben, C. N. Borca, S. Adenwalla, A. V. Bune, S. Ducharme, V.M. Fridkin, S. P. Palto, and N. Petukhova, Phys. Rev. B 59 (1999), p. 1819.

[5] H. Ishii, K. Sugiyama, E. Ito, and K. Seki, Adv. Mater. 11 (1999), p. 605 .

[6] X. Y. Zhu, Surf. Sci. Rep. 56 (2004), p. 1.

[7] S. Balaz, A. N. Caruso, N. P. Platt, D. I. Dimov, N. M. Boag, J. I. Brand, Ya. B. Losovyj, and P. A. Dowben, J. Phys. Chem. B 111 (2007), p. 7009 and the references therein.

[8] S. Kera, Y. Yabuuchi, H. Yamane, H. Setoyama, K. K. Okudaira, A. Kahn, and N. Ueno, Phys. Rev. B 70 (2004), p. 085304.

[9] M. Fahlman, A. Crispin, X. Crispin, S. K. M. Henze, M. P. de Jong, W. Osikowicz, C. Tengstedt, and W.R. Salaneck, J. Phys. Condens. Matter 19 (2007), p. 183202.

[10] L. Ramoino, M. von Arx, S. Schintke, A. Baratoff, H. J. Guntherodt, and T. A. Jung, Chem. Phys. Lett. 417 (2006), p. 22.

[11] L. M. Blinov, V. M. Fridkin, S. P. Palto, A. V. Bune, P. A. Dowben, and S. Ducharme, Uspekhi Fizicheskikh Nauk 170 (2000), p. 247 (Russian edition).

L. M. Blinov, V. M. Fridkin, S. P. Palto, A. V. Bune, P. A. Dowben, and S. Ducharme, Physics Uspekhi 43 (2000), p. 243 (English edition).

[12] B. Xu, Y. Ovchenkov, M. Bai, A. N. Caruso, A. V. Sorokin, S. Ducharme, B. Doudin, and P. A. Dowben, Appl. Phys. Lett. 81 (2002), p. 4281.

[13] J. Xiao, A. Sokolov, and P. A. Dowben, Appl. Phys. Lett. 90 (2007), p. 242907.

[14] R. C. G. Naber, C. Tanase, P. W. M. Blom, G. H. Gelinck, A. W. Marsman, F. J. Touwslager, S. Setayesh, and D. M. De Leeuw, Nat. Mater. 4 (2005), p. 243.

[15] G. H. Gelinck, A. W. Marsman, F. J. Touwslager, S. Setayesh, D. M. De Leeuw, R. C. G. Naber, and P. W. M. Blom, Appl. Phys. Lett. 87 (2005), p. 092903.

[16] S. H. Lim, A. C. Rastogi, and S. B. Desu, J. Appl. Phys. 96 (2004), p. 5673 .

[17] T. J. Reece, S. Ducharme, A. V. Sorokin, and M. Poulsen, Appl. Phys. Lett. 82 (2003), p. 142.

[18] R. V. Plank, N. J. DiNardo, and J. M. Vohs, Chem. Phys. Lett. 263 (1996), p. 33.

[19] J. Choi, M. Chipara, B. Xu, C. S. Yang, B. Doudin, and P. A. Dowben, Chem. Phys. Lett. 343 (2001), p. 193.

[20] B. Xu, J. Choi, and P. A. Dowben, J. Vac. Sci. Technol. A 20 (2002), p. 741.

[21] B. Xu, J. Choi, A. N. Caruso, and P. A. Dowben, Appl. Phys. Lett. 80 (2002), p. 4342.

[22] H. Yamane, Y. Yabuuchi, H. Fukagawa, S. Kera, K. K. Okudaira, and N. Ueno, J. Appl. Phys. 99 (2006), p. 093705.

[23] M. Gorgoi and D. R. T. Zahn, J. Phys. IV (France) 132 (2006), p. 337.

[24] L. Lozzi, S. Santucci, S. La Rosa, and S. Picozzi, J. Chem. Phys. 121 (2004), p. 1883.

[25] J. E. Downes, C. McGuinness, P. A. Glans, T. Learmonth, D. Fu, P. Sheridan, and K. E. Smith, Chem. Phys. Lett. 390 (2004), p. 203. 
[26] M. L. M. Rocco, K. H. Frank, P. Yannoulis, and E. E. Koch, J. Chem. Phys. 93 (1990), p. 6859.

[27] X. Lu, K. W. Hipps, X. D. Wang, and U. Mazur, J. Am. Chem. Soc. 118 (1996), p. 7197.

[28] R. Madru, G. Guillaud, M. Sadoun, M. Maitrot, J. J. Andre, J. Simon, and R. Even, Chem. Phys. Lett. 145 (1988), p. 343.

[29] M. Maitrot, G. Guillaud, B. Boudjema, J. J. Andre, H. Strzelecka, J. Simon, and R. Even, Chem. Phys. Lett. 133 (1987), p. 59.

[30] J.-W. Wang, Q.-D. Shen, C.-Z. Yang, and Q.-M. Zhang, Macromolecules 37 (2004), p. 2294.

[31] J.-W. Wang, Q.-D. Shen, H.-M. Bao, C.-Z. Yang, and Q.-M. Zhang, Macromolecules 38 (2005), p. 2247.

[32] J. Xiao, L. G. Rosa, M. Poulsen, D. Q. Feng, S. Reddy, J. M. Takacs, L. Cai, J. Zhang, S. Ducharme, and P. A. Dowben, J. Phys. Condens. Matter 18 (2006), p. L155.

[33] H. Qu, W. Yao, T. Garcia, J. Zhang, A. V. Sorokin, S. Ducharme, P. A. Dowben, and V. M. Fridkin, Appl. Phys. Lett. 82 (2003), p. 4322.

[34] L. Cai, H. Qu, C. Lu, S. Ducharme, P. A. Dowben, and J. Zhang, Phys. Rev. B 70 (2004), p. 155411.

[35] Ya. Losovyj, I. Ketsman, E. Morikawa, Z. Wang, J. Tang, and P. Dowben, Nucl. Instrum. Methods Phys. Res. A 582 (2007), p. 264.

[36] J. Choi, P. A. Dowben, S. Ducharme, V. M. Fridkin, S. P. Palto, N. Petukhova, and S. G. Yudin, Phys. Lett. A 249 (1998), p. 505.

[37] J. Choi, C. N. Borca, P. A. Dowben, A. Bune, M. Poulsen, S. Pebley, S. Adenwalla, S. Ducharme, L. Robertson, V. M. Fridkin, S. P. Palto, N. Petukhova, and S. G. Yudin, Phys. Rev. B 61 (2000), p. 5760 .

[38] C. G. Duan, W. N. Mei, J. R. Hardy, S. Ducharme, J. Choi, and P. A. Dowben, Europhys. Lett. 61 (2003), p. 81.

[39] P. A. Dowben, J. Choi, E. Morikawa, and B. Xu In: H. S. Nalwa, Editor, Characterization and Spectroscopy of Thin Films, vol.
2: Handbook of Thin Films, Academic Press (2002), pp. 61-114 (Chapter 2).

[40] C. N. Borca, T. Komesu, and P. A. Dowben, J. Electron Spectrosc. Relat. Phenom. 122 (2002), p. 259.

[41] G. Greczynski, N. Johansson, M. Lögdlund, L. A. A. Pettersson, W. R. Salaneck, L. E. Horsburgh, A. P. Monkman, D. A. dos Santos, and J. L. Brédas, J. Chem. Phys. 114 (2001), p. 4243.

[42] A. B. Kaiser, Rep. Prog. Phys. 64 (2001), p. 1.

[43] H. Peisert, T. Schwieger, J. M. Auerhammer, M. Knupfer, M. S. Golden, J. Fink, P. R. Bressler, and M. Mast, J. Appl. Phys. 90 (2001), p. 466.

[44] J. P. Velev, C.-G. Duan, K. D. Belashchenko, S. S. Jaswal, and E. Y. Tsymbal, Phys. Rev. Lett. 98 (2007), p. 137201.

[45] E. Y. Tsymbal and H. Kohlstedt, Science $\mathbf{3 1 3}$ (2006), p. 181.

[46] W. F. Brinkman, R. C. Dynes, and J. M. Rowell, J. Appl. Phys. 41 (1970), p. 1915.

[47] J. Rodríguez Contreras, Ferroelectric Tunnel Junctions, Dissertation der Universität zu Köln, Eigenverlag Forschungszentrum Jülich, Jülich, 2004.

J. Rodriguez Contreras, Ferroelectric Tunnel Junctions, ISBN13: 9783893363681, January 2004.

[48] J. Rodríguez Contreras, H. Kohlstedt, U. Poppe, R. Waser, C. Buchal, and N.A. Pertsev, Appl. Phys. Lett. 83 (2003), p. 4595.

[49] R. Meyer and R. Waser, J. Appl. Phys. 100 (2006), p. 051611.

[50] H. Kohlstedt, N. A. Pertsev, J. Rodríguez Contreras, and R. Waser, Phys. Rev. B 72 (2005), p. 125341.

[51] Y. D. Xia, W. Y. He, L. Chen, X. K. Meng, and Z. G. Liu, Appl. Phys. Lett. 90 (2007), p. 022907.

[52] H. Akazawa, Jpn. J. Appl. Phys. 46 (2007), p. L848.

[53] M. P. Singh, L. Méchin, W. Prellier, and M. Maglione, Appl. Phys. Lett. 89 (2006), p. 202906. 